PAPER

\title{
Objective measures for evaluating tonal balance of sound fields
}

\author{
Daiji Takahashi ${ }^{1, *}$, Kanta Togawa ${ }^{2}$ and Tetsuo Hotta ${ }^{3}$ \\ ${ }^{1}$ Department of Urban and Environmental Engineering, Kyoto University, \\ Katsura, Nishikyo-ku, Kyoto, 615-8540 Japan \\ ${ }^{2}$ FUJITEC Co., Ltd., Hikone, 522-8588 Japan \\ ${ }^{3}$ YAMAHA Corpation, Hamamatsu, 430-8650 Japan
}

(Received 16 April 2007, Accepted for publication 10 July 2007)

\begin{abstract}
The purpose of this study is to derive objective measures that can accurately represent the characteristics of a sound field regarding the tonal balance corresponding to our sense of hearing. Two types of listening test were conducted in the form of paired comparison, in which subjects were tested using sound fields produced by convoluting anechoic music sources with impulse responses. In the first listening tests, impulse responses were calculated theoretically for a simple structure of sound field consisting of a direct sound and reflections, and in the second tests, impulse responses were measured at various seats of concert halls. In the latter case, impulse responses that give almost the same distinctness were used in the listening tests. From this investigation, it is found that one objective measure named deviation of level $(D L)$ is a possible effective measure for evaluating the tonal balance of sound fields. The index $D L$ is calculated from data based on the logarithmic scale in both the frequency and magnitude.
\end{abstract}

Keywords: Tonal quality, Objective measure, Spectrum, Listening test, Paired comparison

PACS number: 43.55.Hy, 43.66.Yw [doi:10.1250/ast.29.2]

\section{INTRODUCTION}

Tonal quality is one of the key factors involved in the evaluation of a sound field. Typical examples related to tonal quality are the problem of coloration and the tonal balance of frequency characteristics. Cases where the former becomes a problem are very few. However, there are many cases where the latter becomes a serious problem, for example, the insufficiency of bass sounds and/or the lack of treble sounds. The main causes of these problems are the 'seat dip effect' and the interference of reflections, among others. If there is no appropriate index, even though the cause is specified, it is almost impossible to establish the level of the problem, not to mention find a solution.

The related objective measures proposed up to now have been 'bass ratio' [1] in relation to 'acoustical warmth' and 'bass level balance' [2,3]. These indexes, however, do not show good correlation with the perception of bass [3]. Soulodre and Bradley [4] proposed an index 'early bass level' for the evaluation of bass sound. Although the index shows good performance, it includes the parameter of the overall strength of the sound (loudness). It seems reasonable that the richness of both bass and treble sounds

*e-mail: tkhs@archi.kyoto-u.ac.jp depends on loudness. In this point, the index 'early bass level' is different from the concept of the present study in terms of tonal quality.

In this study, the tonal balance of sound fields independent of the overall strength of the sound is considered and an objective measure corresponding to the perception of this quality is proposed. Two types of subjective experiment are conducted and the results are discussed in relation to this new objective measure.

\section{PROPOSED OBJECTIVE MEASURE}

The poor tonal quality of sound fields is usually perceived in terms of the insufficiency of bass sounds and/ or the lack of treble sounds. In both cases, however, the problem originates from the change in the spectrum. Thus, it is desirable to derive a single measure for evaluating the tonal quality of sound fields.

In this study, considering the problem of tonal balance, an objective measure is newly developed under the following basic assumptions:

1. Tonal balance is a key factor for evaluating the tonal quality of sound fields.

2. Orchestral music with instruments across the whole frequency range is the most appropriate sound for evaluating the tonal balance. 

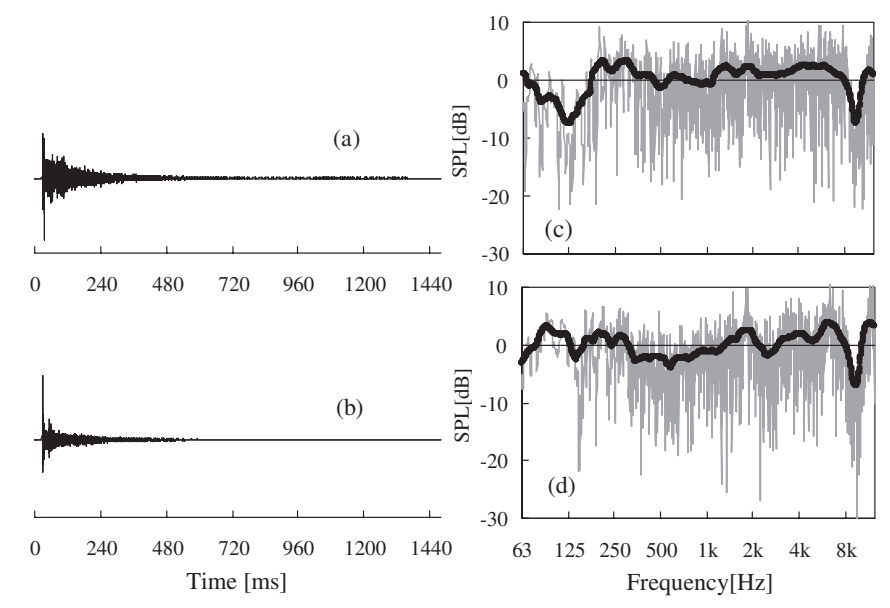

Fig. 1 Examples of impulse response (a), (b) and spectrum (c), (d) measured at different seats of a concert hall of 500 seats.

3. In determining the tonal balance of the orchestral sound, the conductor's judgment is the best.

All information related to the tonal quality at a receiving point is included in the impulse response at the point. One example of this concept is shown in Fig. 1. The frequency characteristics can be obtained from the impulse response by taking the Fourier transform. In this process, the data of the spectrum are calculated as discrete values with equal intervals $\Delta f$ on the frequency axis as follows:

$$
\Delta f=f_{\mathrm{S}} / N,
$$

where $f_{\mathrm{S}}$ is the sampling frequency and $N$ is the number of samples. The sampling frequency is $44.1 \mathrm{kHz}$ throughout this study. The performance of the objective measure for evaluating tonal balance depends on how the parameters appearing in this spectrum are manipulated. Regarding this matter the following three items should be considered:

1. What frequency range should be used for extracting the index?

2. Is the variable $\Delta f$, which linearizes the index with respect to frequency, appropriate or not as a parameter of the index?

3. Which is more suitable when considering the magnitude of the spectrum, a logarithmic scale or a linear scale?

For item 2, from well-known findings concerning the perception of pitch, it seems to be appropriate to use data at equal intervals on the logarithmic scale of frequency, and for item 3 , there seems to be no problem with using the $\mathrm{dB}$ value, which is widely accepted as having a good response to loudness.

As regards item 1, listening tests were carried out to specify the frequency range that corresponds to the perception of the tonal variation of sounds. All listening tests including those described in Sections 3 and 4 were
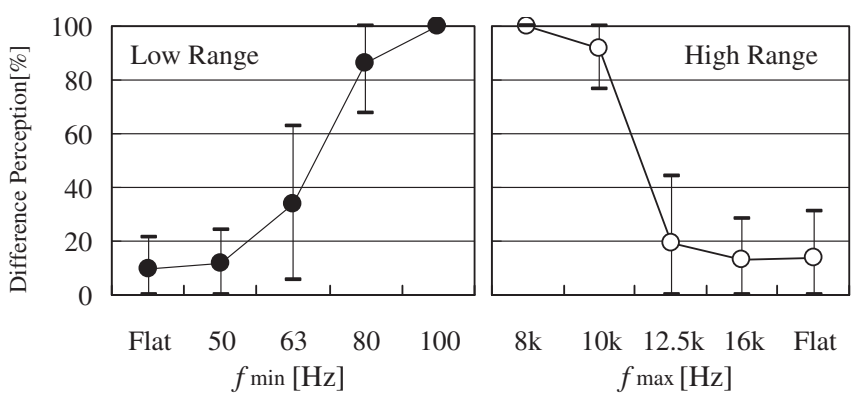

Fig. 2 Results of difference perception with the variation of the frequency range.

conducted using headphones with an equalized response, and with music samples in stereo, in which the volume of the music stimuli were adjusted to the subject's preference. The details of equalizing the headphones are described in Section 3. Five subjects having normal hearing were used in this experiment. The results of the difference perception are plotted with respect to the limits of frequency, $f_{\min }$ and $f_{\max }$, in Fig. 2, which are obtained from the data of subjective paired-comparison tests. The subjects are asked whether or not a difference is perceived between the filtered and the bypassed music sources. The percentage of the judgments in which a difference was detected was statistically analyzed. The results of the $95 \%$ confidence interval for the perception of the difference are shown in these graphs, in which a circle represents the mean value. The music source is the overture of 'Le Nozze di Figaro' (Mozart) and the high- and low-pass filters used are shown in Fig. 3.

From the results for a difference perception of $50 \%$, it seems reasonable to adopt the range $63-12.5 \mathrm{kHz}$, which is used for calculating the index given in Eq. (2). The spectrum of the music source is shown in Fig. 4 (for the case of Mozart), in which a tendency similar to that of the difference perception at low frequencies can be seen. To confirm this observation, the spectra of other music sources are also shown in this figure, which are taken from anechoic music samples recorded on CD [5]. These music

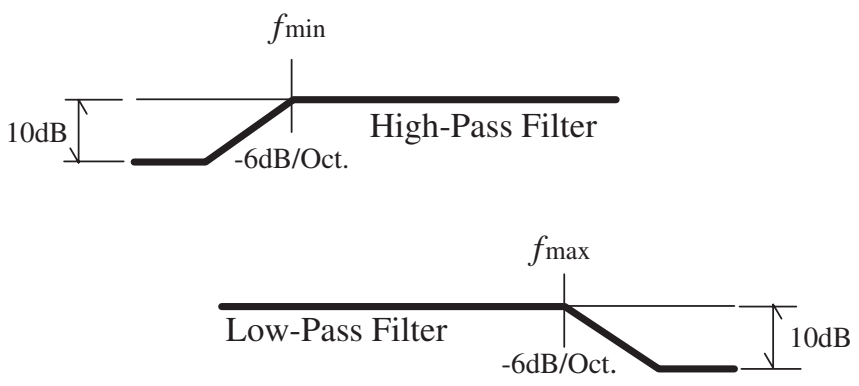

Fig. 3 High- and low-pass filters used in experiment for detecting the effective frequency range. 


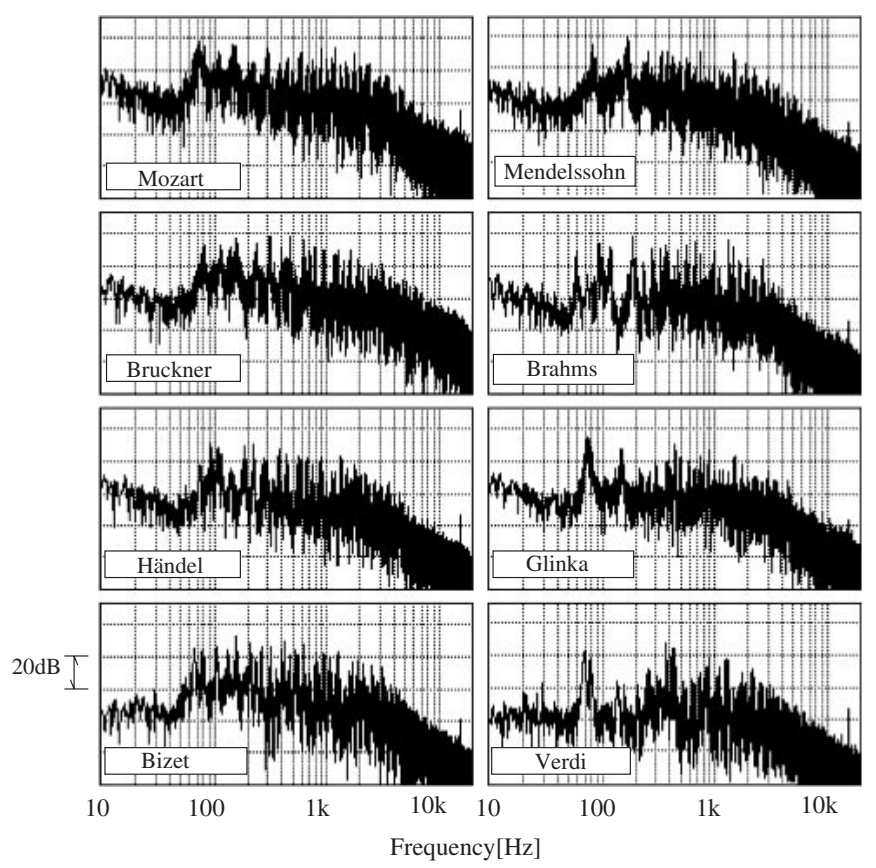

Fig. 4 Spectra of anechoic music samples, Mozart: 'Le Nozze di Figaro', Mendelssohn: Symphony No. 3 'Scottish,' Bruckner: Symphony No. 4 in E flat major, Brahms: Symphony No. 4 in E minor, Händel: 'Water Music,' Glinka: 'Ruslan and Ljudmila,' Bizet: 'L'Arlesienne,' Verdi: 'La Traviata.'

samples are chosen in accordance with item 2 of the basic assumptions. Almost the same tendency, particularly at low frequencies, can be seen in each spectrum. From these results together with the data for the frequency ranges of standard musical instruments [6], the frequency range 63$12.5 \mathrm{kHz}$ seems to be reasonable for evaluating the tonal balance.

From the discussion above, the following index named $D L$ (the standard deviation of the spectrum level of the impulse response) is proposed as a measure for evaluating the tonal balance:

$$
D L=\sqrt{\frac{\sum_{i=1}^{N}\left(S P L_{i}-\mu\right)^{2}}{N}}, \quad \mu=\frac{1}{N} \sum_{i=1}^{N} S P L_{i},
$$

where $S P L_{i}$ is the sound pressure level[dB] sampled at any frequency interval of the data determined by the moving average of the raw data (discrete data with linear interval $\Delta f$ ). The moving average is taken over the frequency range of interest, which is from $f_{\min }$ to $f_{\max }$. One example of the averaged data is shown in Fig. 5, in which black circles denote the data obtained from the moving average (bandwidth of $1 / 3$ octave and the shift used in averaging is $1 / 12$ octave). The values of the parameters in the moving average, however, may have a large effect on the measure's performance, which will be discussed in the following section.

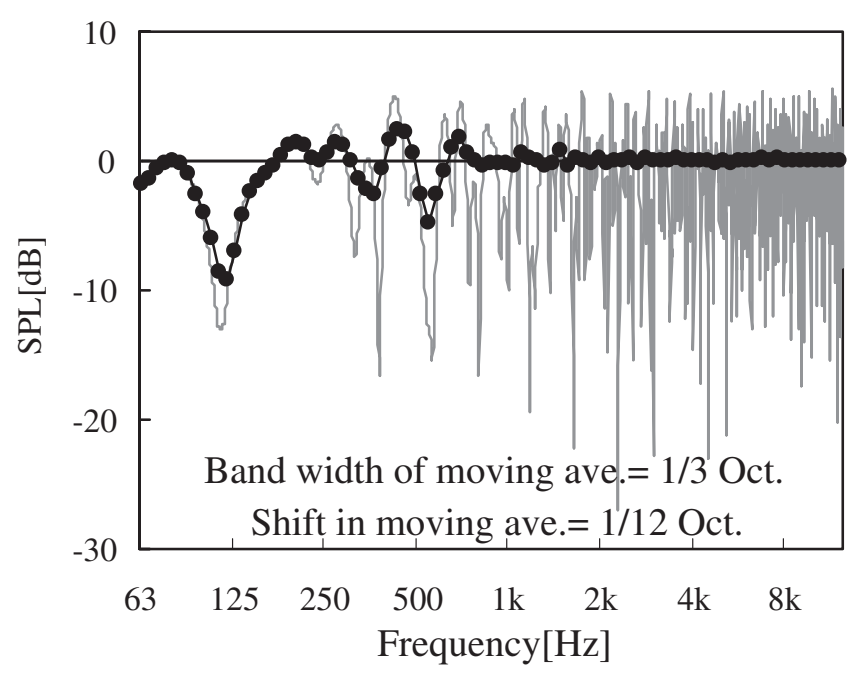

Fig. 5 Data used in calculation of the index $D L$.

\section{EXPERIMENTS USING SIMULATED SOUND FIELDS}

To find appropriate values of the parameters (bandwidth and shift interval in moving average), listening tests were conducted using impulse responses calculated theoretically under the condition that surfaces met at right angles (assuming an open-air theater). The configuration of the model is shown in Fig. 6, and six types of impulse response, and the spectrum for each type calculated using the model with various parameters are shown in Fig. 7. These parameters, listed in Table 1, are source locations $d_{1}$ and $d_{2}$, receiver location $R$, horizontal angle $\varphi$ and elevation angle $\theta$. Figure 8 shows the outline of the experiments, in which two anechoic music samples, the overture of 'Le Nozze di Figaro'(Mozart) and Symphony No. 3, 'Scottish'(Mendelssohn), were used. Each signal segment lasting $10 \mathrm{~s}$ was arranged to obtain a series of comparison pairs. Using headphones, subjects were pre-

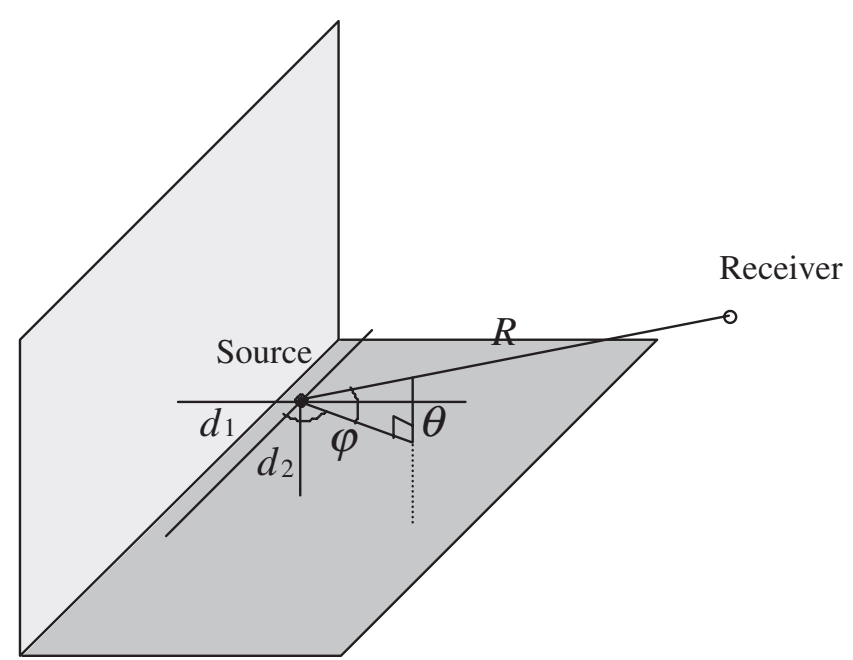

Fig. 6 Simulation model for calculating the impulse response. 


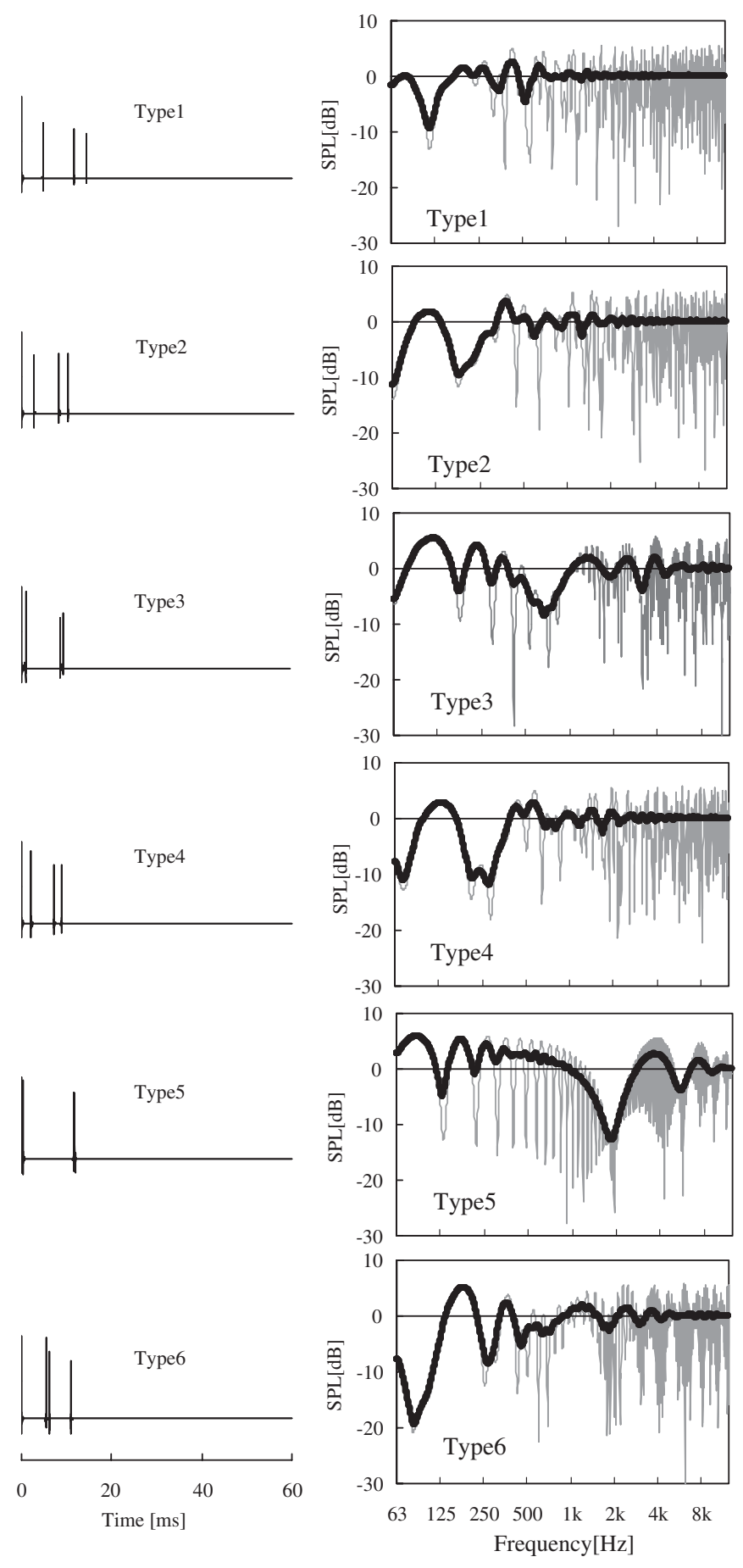

Fig. 7 Impulse response and spectrum of the simulated sound fields.

sented with sounds produced by convoluting these sources with impulse responses. A nonfiltered sample was included, which is expressed as Type 0 . To minimize the effects of differences in loudness, energy of each presented stimulus was equalized, and the volume was adjusted to the subject's preference. The number of subjects for both this experiment and the experiment described in Section 4 was 12. All test subjects had normal hearing, and half of the subjects had experience playing an instrument in an orchestra. The frequency response at the entrance of the ear
Table 1 Parameters used in the simulation for calculating the impulse response.

\begin{tabular}{rrcccc}
\hline & $R[\mathrm{~m}]$ & $d_{1}[\mathrm{~m}]$ & $d_{2}[\mathrm{~m}]$ & $\theta\left[^{\circ}\right]$ & $\varphi\left[^{\circ}\right]$ \\
\hline Type 1 & 4.9 & 2.0 & 1.5 & 17 & 85 \\
Type 2 & 7.4 & 1.6 & 1.0 & 20 & 60 \\
Type 3 & 5.3 & 1.5 & 0.5 & 10 & 75 \\
Type 4 & 6.8 & 1.5 & 0.5 & 40 & 75 \\
Type 5 & 20.7 & 2.0 & 1.0 & 0 & 75 \\
Type 6 & 18.5 & 1.5 & 1.5 & 40 & 45 \\
\hline
\end{tabular}

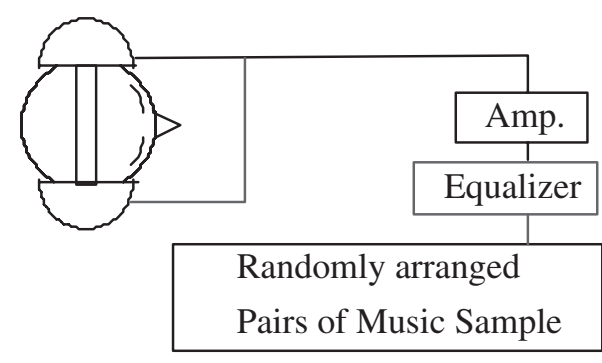

Fig. 8 Outline of the subjective experiments.

canal reproduced by the headphones was equalized using an equalizer to the state at which the characteristics were the same as those of the response at the same place due to sounds emitted from a loudspeaker with flat characteristics in an anechoic chamber. In the case of using a loudspeaker, the response at the ear canal depends on the angle of incidence, which was here taken to be an averaged value of the response at $0^{\circ}$ (front face), $45^{\circ}, 90^{\circ}, 135^{\circ}$ and $180^{\circ}$ (back face) in the horizontal plane.

All tests were conducted in the form of paired comparisons using Scheffe's paired comparison method. The following instructions were given prior to the experiments. "Good tonal balance means that the bass sound and treble sound are appropriate in the total frequency characteristics. How do you evaluate the latter music sample compared with the former one?" Subjects were asked to indicate one evaluation of each sample on a scale from -2 to +2 .

The psychological judgments were extracted from the evaluations by statistical analysis. Figures 9 and 10 show the correlation between the objective measure $D L$ and the subjective score in terms of tonal balance. A higher score represents a higher evaluation of the sample, and $Y S$ (yardstick) denotes the critical value indicating a significant difference (95\% confidence). $R^{2}$ shows the coefficient of determination. The parameter of the bandwidth in the moving average is taken to be $1 / 6,1 / 3$, and 1 octave. Relatively good correlation between the objective measure and the subjective response can be seen in these cases $\left(D L_{-} 1 / n-\log \right)$. For reference, the results of the cases in which $S P L_{i}$ was obtained from the data with a linear frequency interval ( $D L$ Raw and $D L_{-} 1 / 3$-Lin.) are also 

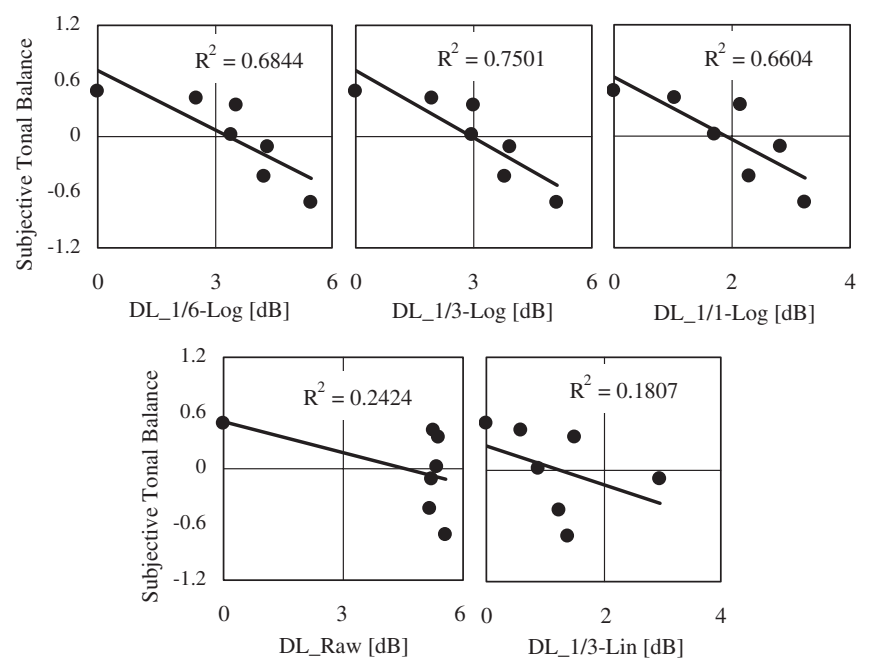

Fig. 9 Effect of bandwidth used in averaging for the case of 'Figaro', with $Y S=0.315$.
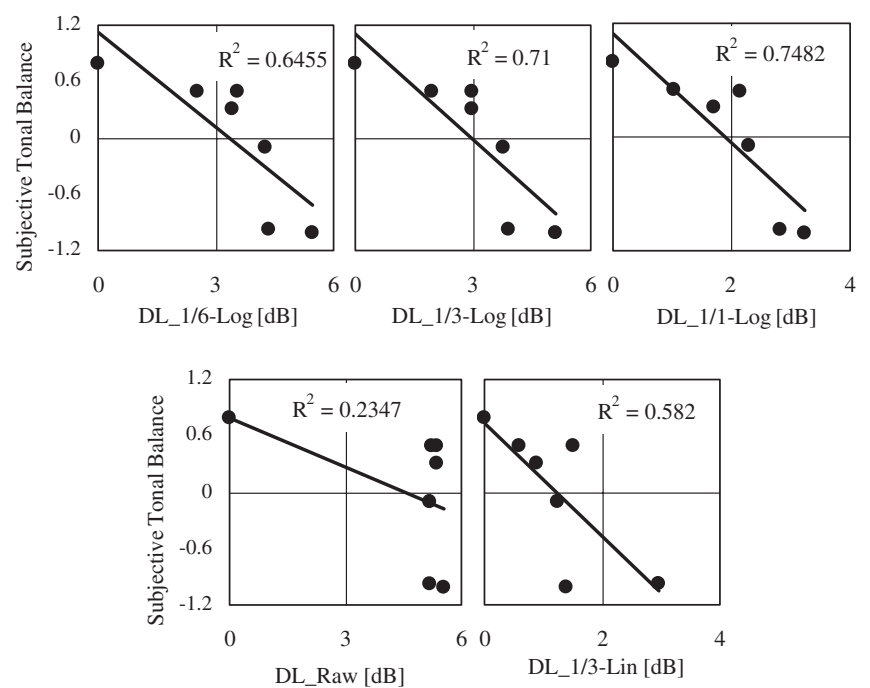

Fig. 10 Effect of bandwidth used in averaging for the case of 'Scottish,' with $Y S=0.282$.

shown in this graph, which show relatively poor correlation with the subjective score. The effects of the shift in moving average are shown in Table 2. From these results, it is seen that a shift interval of $1 / 12$ octave or less causes a difference of less than $\pm 0.1 \mathrm{~dB}$ for $D L$. In this experiment, as shown in Figs. 10 and 11, the highest evaluation is given to Type 0 , which is the case of a nonfiltered music source having $D L=0$. The validity of the third assumption given in Section 2 may be confirmed from this fact.

In summary, from experiments using a simulation of sound reflections, an objective measure $D L$ calculated from the moving average of data with the parameters of $1 / 3$ octave bandwidth and 1/12-1/48 octave shift interval shows a good correlation with the subjective response in terms of tonal balance.
Table 2 Effects of shift in moving average on the value of $D L$.

\begin{tabular}{lccccc}
\hline & \multicolumn{5}{c}{ Shift in $1 / n$-Octave Interval } \\
\cline { 2 - 6 } & $1 / 48$ & $1 / 24$ & $1 / 12$ & $1 / 6$ & $1 / 3$ \\
\hline Type 1 & 1.95 & 1.94 & 1.94 & 1.91 & 1.79 \\
Type 2 & 2.96 & 2.98 & 3.02 & 3.12 & 3.24 \\
Type 3 & 2.99 & 2.99 & 3.00 & 3.01 & 2.98 \\
Type 4 & 3.78 & 3.79 & 3.80 & 3.82 & 3.61 \\
Type 5 & 3.92 & 3.92 & 3.93 & 3.93 & 3.89 \\
Type 6 & 5.12 & 5.11 & 5.12 & 5.13 & 5.16 \\
\hline
\end{tabular}
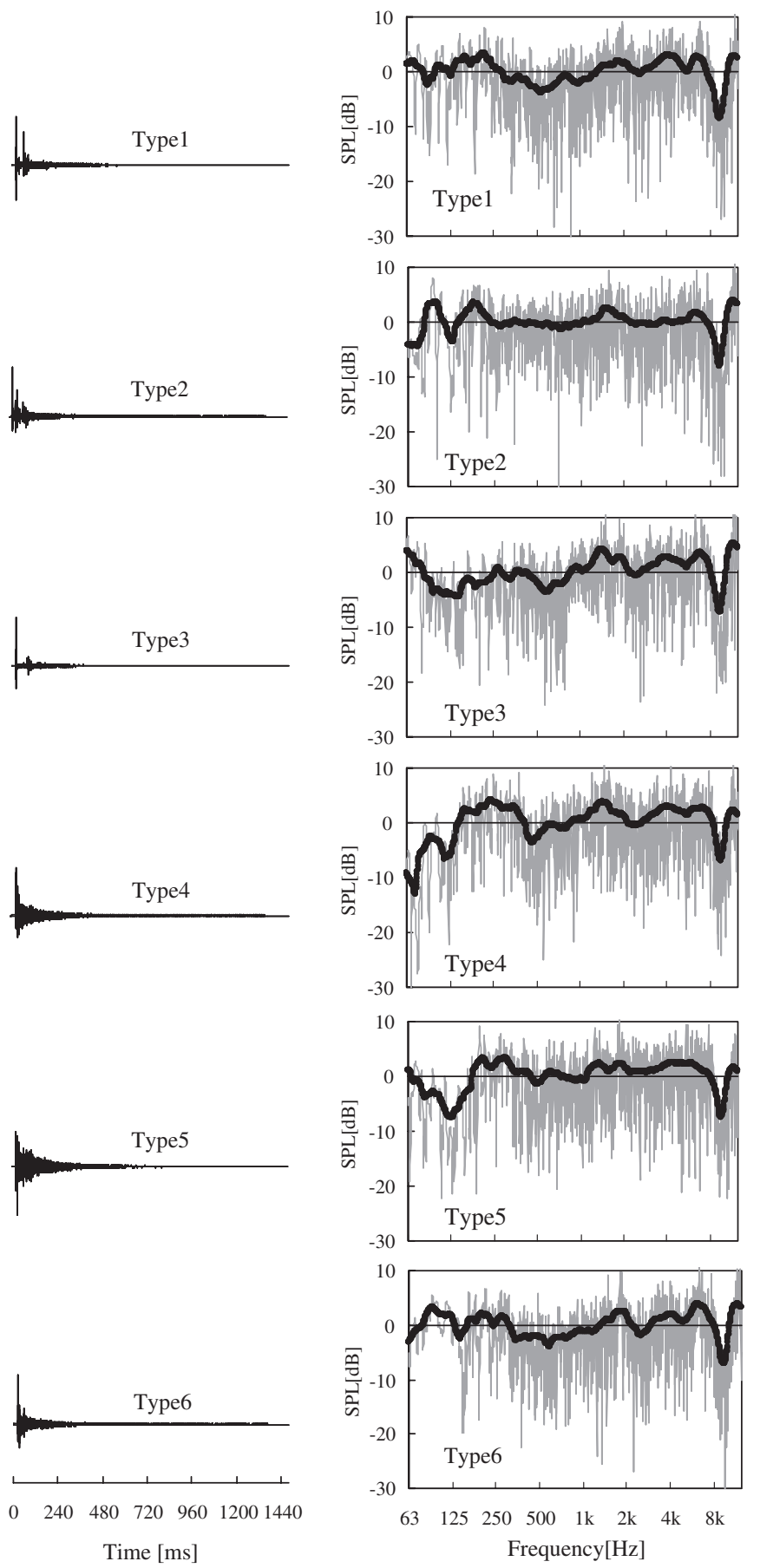

Fig. 11 Impulse response and spectrum measured in concert halls. 
Table 3 Values of some objective measures for the time structure of the response.

\begin{tabular}{lccccc}
\hline & $R T[\mathrm{~s}]$ & $E D T[\mathrm{~s}]$ & $C[\mathrm{~dB}]$ & $t_{\mathrm{s}}[\mathrm{ms}]$ & $S T I$ \\
\hline Type 1 & 2.21 & 2.13 & 0.0 & 137.42 & 0.49 \\
Type 2 & 2.21 & 1.99 & 1.49 & 107.29 & 0.53 \\
Type 3 & 2.22 & 2.06 & 1.41 & 107.51 & 0.52 \\
Type 4 & 1.59 & 1.57 & 0.83 & 102.45 & 0.56 \\
Type 5 & 1.59 & 1.47 & 0.75 & 104.19 & 0.52 \\
Type 6 & 1.62 & 1.71 & -0.69 & 116.04 & 0.54 \\
\hline
\end{tabular}

\section{EXPERIMENTS USING HALL SOUND FIELDS}

In this section, the possibility of applying $D L$ to actual sound fields is discussed by conducting the same subjective tests as those discussed in the previous section. In the experiment, six types of impulse response (Types 1 to 6 ) were selected from many data measured in two concert halls. In preliminary investigations, it was found that extreme differences in the time structure of the response affected the results from subjective experiments, for example, the case of the response measured at a central receiving point in front of the stage, where the source is near and the efficient reflecting surfaces are far from the point, and the case when sound is very clear (high distinctness). Each impulse response and its spectrum is shown in Fig. 11, and for reference, the objective parameters used in the time structure of the response are given in Table 3. These test samples were chosen with reference to the values of clarity index $C$, center time $t_{\mathrm{s}}$ and $S T I$. Incidentally, there was a place where these values were $3.01,80.59$ and 0.613 , respectively, while the values of $R T$ and $E D T$ were 2.11 and 1.92, respectively. This place is in the same concert hall as the place where samples of Types 1, 2 and 3 were recorded. The subjective experiments were carried out, and the data were analyzed in the same way as in Section 3.

While calculating the objective measure $D L$, care is required in selecting the time length (the time window in the FFT process) for calculating the spectrum. This seems to be related to our subjective time length for the perception of the tonal quality of sound, and does not appear in the case of the simulated response in Section 3, which is composed of only four reflections, as shown in Fig. 7. An example of the difference for Type 6 is shown in Fig. 12. The calculated value of $D L$ is $3.0 \mathrm{~dB}$ in the time window $0-80 \mathrm{~ms}$ and $D L=2.1 \mathrm{~dB}$ for the total time $(0-$ $1,440 \mathrm{~ms})$. To specify an appropriate value of the time window, the relation between $D L$ and the score of subjective tonal balance for various time windows is discussed. Some examples of the relation (for the case of 'Figaro') are shown in Fig. 13. The results show that the time window of $320 \mathrm{~ms}$ gives a good correlation. The

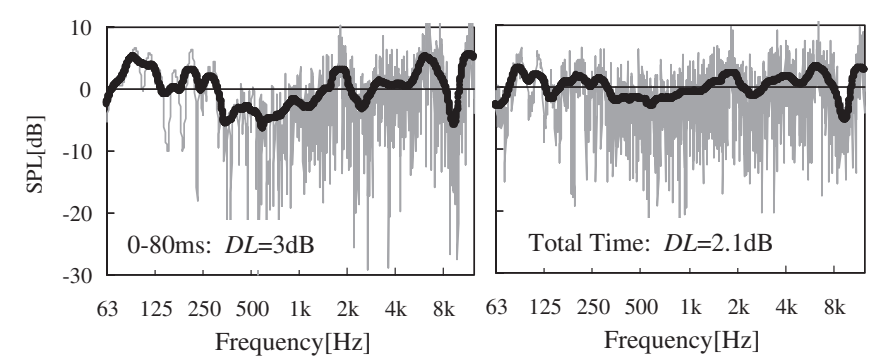

Fig. 12 Example of the effect of time window on the index $D L$.
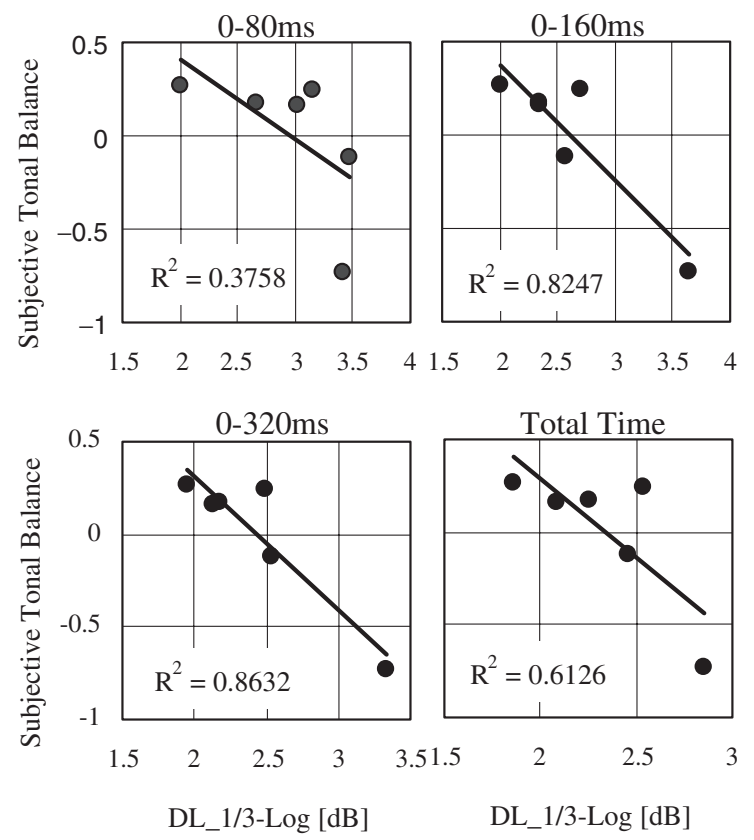

Fig. 13 Effect of time window on the calculation of $D L$ from the data for the case of 'Figaro,' with $Y S=0.319$.

results of a more detailed investigation of the data obtained using music samples of 'Figaro' and 'Scottish' are shown in Fig. 14, in which the coefficient of determination $R^{2}$ is plotted as a function of the time window. From the results described above, a time window of $200-400 \mathrm{~ms}$ is recommended for the calculation of $D L$. The time window of the spectrum in Fig. 11 is $0-300$ ms. Figure 15 shows the correlation between the subjective score and the objective measure $D L$ for the perception of tonal balance for 'Figaro' and 'Scottish.' From these results, the bandwidth of $1 / 6$ octave in the moving average shows a strong correlation with the subjective response. However, the significant difference for each type is rather low in comparison with the results shown in Figs. 9 and 10. Therefore, considering all our results, it may be concluded that the adoption of a bandwidth of $1 / 3$ octave for averaging is appropriate.

In Fig. 15, an extremely poor evaluation with the highest value of $D L$ is given to Type 4 (for both 'Figaro' and 'Scottish'), and the highest evaluations are given to 


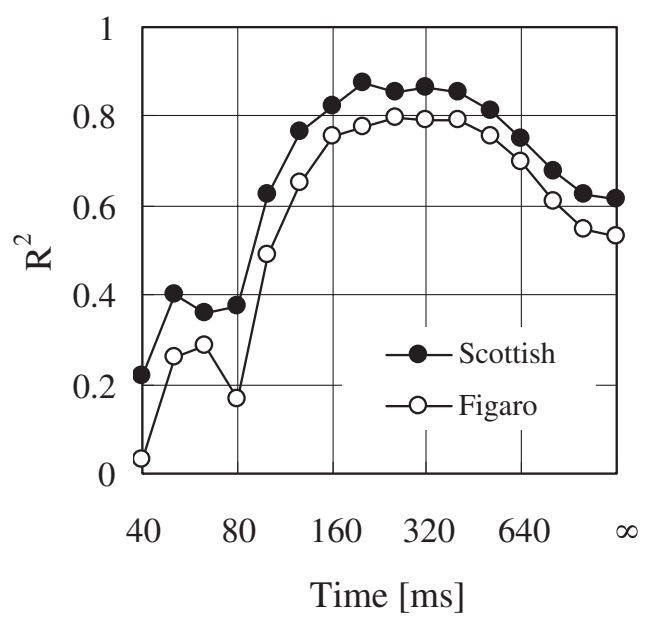

Fig. 14 Effect of time window on the performance of $D L$ from the data for the cases of 'Figaro' and 'Scottish.'
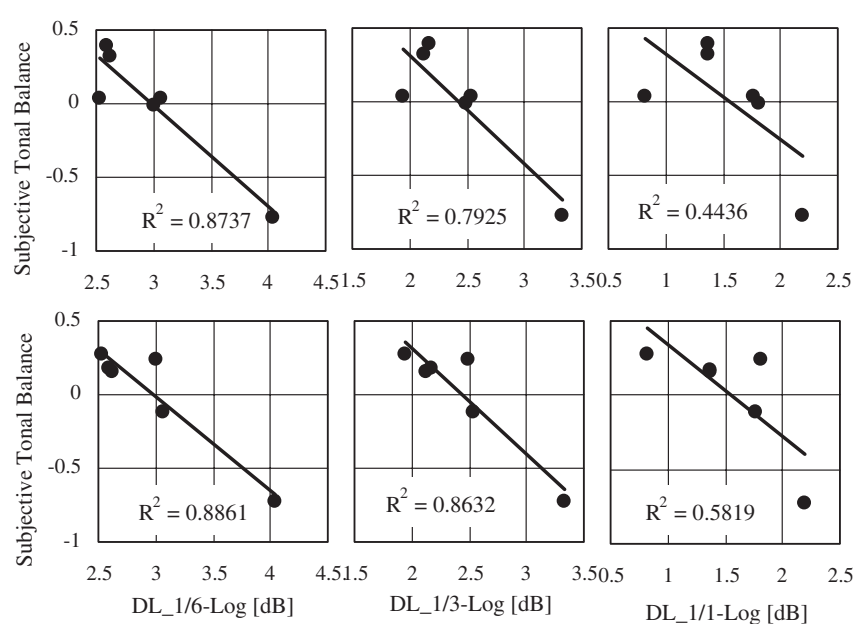

Fig. 15 Result of the experiment using actual hall response from the data for the cases of 'Figaro' with $Y S=0.319$ in the upper row and 'Scottish' with $Y S=0.315$ in the lower row.

Type 1 (for 'Figaro') and Type 2 (for 'Scottish'). As can be seen in the corresponding spectrum in Fig. 11, in comparison with other types, the spectrum of Type 4 is relatively rough, particularly at low frequencies, and the highest evaluation is given to a spectrum with relatively smooth characteristics.

\section{CONCLUSIONS}

In this study, an objective measure $D L$ was proposed for evaluating the tonal balance of sound fields, and the effectiveness of the measure was examined by subjective paired comparison tests. The index $D L$ is calculated using the $S P L[\mathrm{~dB}]$ data of the spectrum, at equal intervals on the logarithmic scale of frequency. The procedure for calculating $D L$ is summarized as follows:

1. Measure the impulse response of the sound field.

2. Calculate the spectrum of the impulse response by FFT, in which a time window of $200-400 \mathrm{~ms}$ from the arrival of the direct sound is recommended.

3. Calculate the moving average of the spectrum, in which the bandwidth is $1 / 3$ octave and the shift interval of the frequency on the logarithmic scale is less than $1 / 12$ octave.

Using the data obtained from this procedure, the index $D L$ is calculated from Eq. (2) and can be used as an effective measure for evaluating the tonal balance, which is considered to be one aspect of the tonal quality of the sound field.

\section{REFERENCES}

[1] L. L. Beranek, Music, Acoustics \& Architecture (John Wiley \& Sons, New York, 1962), pp. 435-436.

[2] M. Barron, "Subjective study of British symphony concert halls," Acustica, 66, 1-14 (1988).

[3] M. Barron, "Bass sound in concert auditoria," J. Acoust. Soc. Am., 98, 1088-1098 (1995).

[4] G. A. Soulodre and J. S. Bradley, "Subjective evaluation of new room acoustic measures," J. Acoust. Soc. Am., 98, 294-301 (1995).

[5] T. Hidaka, K. Kageyama and S. Masuda, "Recording of anechoic orchestral music and measurement of its physical characteristics based on the auto-correlation function," Acustica, 67, 68-70 (1988).

[6] M. Barron, Auditorium Acoustics and Architectural Design (E \& FN SPON, London, 1993), pp. 11-13. 